\title{
Application of Artificial Intelligence in detection of diseases in plants: A Survey
}

\author{
Gyan Singh Sujawat ${ }^{a}$, Dr. Jitendra Singh Chouhan ${ }^{b}$ \\ Research Scholar, Department of Computer Science, Sangam University, Bhilwara, Rajasthan, India \\ Professor, Department of Computer Science, ARAVALI Institute of Technical Studies,, UdaipurRajasthan, Indi
}

Article History: Agriculture, Artificial Intelligence, Image Processing, Disease Detection, Machine learning

\begin{abstract}
The agriculture sector can be considered as the backbone for any developing economy. To obtain the maximum yield from the crops, it is required that farmers should be provided with the best technologies and methodologies. Artificial intelligence is having its vast applications in various sectors. Due to its ability to perceive the problems, developing the appropriate reasons for that and to establish optimal solutions for it, artificial intelligence can act as a great aid in addressing the diseases of crops. The paper presents a brief overview of the application of artificial intelligence in agriculture, its available techniques for agriculture and highlights the various methods available for the detection of diseases in crops
\end{abstract}

Keywords: LGS, mathematics, teacher, difficulty

\section{Introduction}

Artificial intelligence has a huge impact in all Industrial Sectors. Lately, Artificial Intelligence (AI) has been progressing at an outstanding speed. AI accomplished solving numerous problems and saving a profitable resource by minimizing environmental deterioration. Artificial Intelligence is making a revolution in agriculture by replacing traditional methods by using methods that are more efficient and helping the world to become a better place [1]. Agriculture is the principal foundation of subsistence for about 58\% of India's population. The population is expanding enormously with this expansion the interest of food and business is likewise expanding. Intervening of AI in Agriculture is serving farmers to recover their farming efficiency and diminish environmental hostile influences [2]. Disease infection is the main drawback of Agriculture. Due to this drawback, the Quality and Quantity of agriculture products are degraded [3]. To identify and detect the disease on agriculture product, the AI technique is introduced. In this paper, we are presenting a survey for application of artificial intelligence in detection of diseases in agriculture.

\section{Artifical Intelligence In Agriculture}

Artificial Intelligence (AI) is one of the mainstream of research in software engineering with its rapid scientific advancement and the tremendous region of Application. The fundamental idea of AI in agriculture is its adaptability, speedy performance, precision, and cost-viability [4]. Artificial Intelligence in Agriculture not only helps farmers to use their farming skills but also shifts to direct farming to get higher yields and better quality with less resources [5]. AI-based technology helps to improve efficiency in all sectors and manages the challenges facing various industries including various sectors in the agricultural sector such as crop harvesting, irrigation, soil content sensitivity, crop monitoring, weed, harvest and establishment. AI technology helps diagnose plant diseases, pests, and malnutrition on farms and AI sensors can detect and identify weeds. The mythologies utilized for disease detection, segmentation of the affected part and classification of the diseases [3]. Artificial Intelligence can offer an effective and practical solution for the problem and introduced Machine Learning (ML) and Deep learning (DL) [6]. Machine learning to train the large data sets available publicly give us a clear way to detect the disease present in plants in a colossal scale [7]. The machine learning-based approaches, which will be used for detecting and classifying the diseases on agricultural products including various plants, fruits and vegetables [8]. A robot that identifies the leaf disease utilizing image processing and Machine learning is conveyed [9]. The survey of CNN-based research efforts applied in the agricultural domain [10]. Detecting diseases and pests from rice plant images using $\mathrm{CNN}$ (convolutional neural networks) [11].

\section{Field Of Artificial Intelligence In Agricultural Sector}

In the field of agriculture, Artificial Intelligence is a rising revolution. Artificial Intelligence has boost crop production and better-quality real-time monitoring, harvesting, processing and marketing.[12]

1. The Internet of things (IoT) driven development 
The Internet-of-Things (IoT) is a foundation to impact a wide array of sectors and industries, ranging from manufacturing, health, communications, and energy to the agriculture industry. The application of IoT in agriculture is about empowering farmers with the decision tools and automation technologies that seamlessly integrate products, knowledge and services for good efficiency, quality, and profit.

\section{Image-based insight generation:}

Drone-based images can help in crop monitoring, scanning of fields and so on. Farmers can join them with PC vision innovation and IOT to guarantee quick activities. These feeds can produce ongoing climate alarms for farmers.

\section{Disease detection:}

The image sensing and analysis make sure that the plant leaf images are segmented into surface areas like background, diseased area and non-diseased area of the leaf. The infected or diseased area is then harvested and sent to the laboratory for additional diagnosis.

\section{ExpertSystem:}

The need for Expert systems for the transfer of technical information in agriculture can be identified by identifying problems through the traditional technology transfer system, and by demonstrating that professional systems can help to overcome the problems identified, and are likely to be improved.

\section{FieldManagement :}

Employing images of high description from the drone and copters systems, real-time estimations can be achieved during the period of cultivation by building a field map and discovering areas where the crops require water, fertilizer and pesticides.

\section{Robotics in Agriculture:}

Agribot or Agbot is an Agriculture Robot. It supports the farmer to increase the crop's efficiency and also reduces the need for manual labour to the farmer. In the upcoming generations, we can expect that these agricultural robots will do the tilling, sowing, harvesting and many other farm works individually. Indeed, even the weeding, ccontrol of pests and diseases will be dealt with by these agricultural robots.

\section{Automation techniques in irrigation and enabling farmers:}

AI accomplished machines alert of historical climate outline, quality of soil and kind of crops to be grown, can automate irrigation and enhance the whole yield. Nearly $70 \%$ of the world's freshwater resource is utilized for irrigation; such automation can conserve water and benefit farmers in managing their water probs.

\section{Crop health monitoring:}

Remote sensing (RS) techniques along with hyperspectral imaging and 3D laser scanning are crucial to constructing crop metrics over thousands of acres of cultivable land.

\section{Application Of Ai Techniques In Agricultural Sector}

\section{Image Processing :}

Image processing is a method, which is used to measure the affected area of disease, and to find differences in the color of the affected area.Thesurveys show detection of disease by using Image processing [3]. Aintroduction a robot in agriculture that detects the leaf disease-using image processing [9].

\section{Machine Learning :}

Machine learning AI Application and have been successfully made in the present world for the diagnosis of diseases.Machine learning algorithms are fast and accurate to detect any diseases. The paperemployed to increase the recognition rate and the accuracy of the results by using machine learning and deep learning algorithm and detect the plant disease [7].The Support Vector Machine (Machine Learning Algorithm) is a better option for detection of diseases [8].

\section{Deep Learning :}

Deep learning helps in finding out a vital relationship in the data as well as it also records the information regarding existing clients that might help patients having similarities in symptom or diseases.Plant disease identification model based on deep learning proposed in this paper can overcome the complexity of the environment and improve the accuracy of identification [13]. 


\section{Convolutional neural networks :}

Convolutional Neural Networks (CNNs) are considered state-of-the-art in image recognition and offer the ability to provide a prompt and definite diagnosis.

\section{Expert System :}

Expert System in the area of agriculture would take the form of Integrated Crop management, decision aids and would encompass irrigation, nutritional disorders and fertilization, weed control, cultivation and herbicidal 6

\section{AI Techniques Used For Detection Of Diseases In Agriculture}

\section{A. Image Processing}

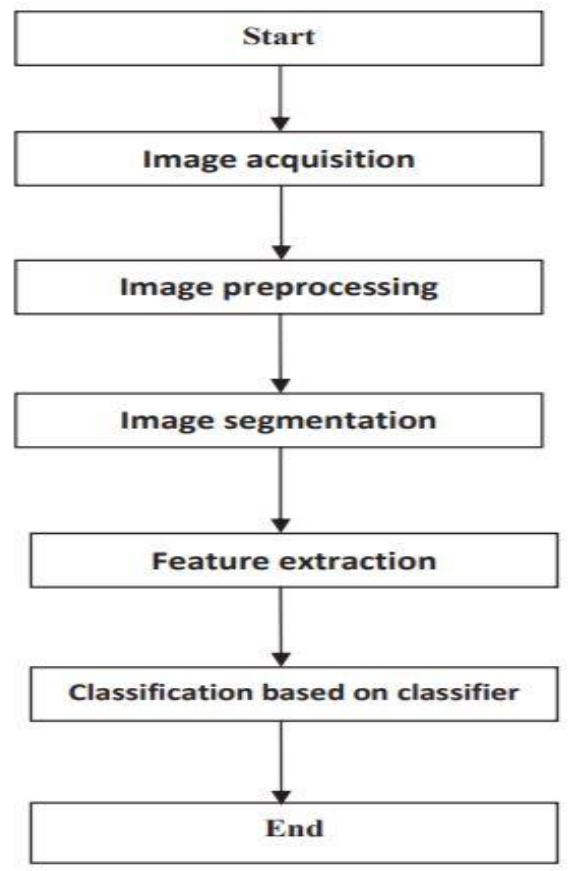

Fig 1: Basic Flowchart Of Disease Detection And Classification[15]

(a) Image Acquisition

Images of the infected leaves are obtained. This database has different types of plant diseases, and the images are stored in JPEG format. These images are then read in MATLAB using the read command.

\section{(b) Image Pre-processing:}

Image pre-processing is used to erase noise from the image or other object exclusion, different pre-processing techniques. Image scaling is used to convert the original image into thumbnails because the pixel size of the original image is large and it requires more time for the overall procedure hence after converting the image into thumbnails the pixel size will get decreases and it will require less time.

\section{(c) Image segmentation:}

Image segmentation is one of the most widely used methods to distinguish pixels of image well in a targeted app. It distributes an image into numerous discrete states such that the pixels have great similarity in each area and high dissimilarity between areas.

\section{(d) Feature Extraction:}

Feature Extraction is an important part of disease detection. It plays an important role in the identification of an object. Feature extraction is utilized in several applications in image processing. Colour, texture edges, morphology are the features, which are utilized in disease detection.

(e) Detection and classification of plant diseases 
The final stages are the detection of the diseases and with the help of disease classify the plants with the disease matches with the given dataset.

\section{B. Convolutional neural networks}

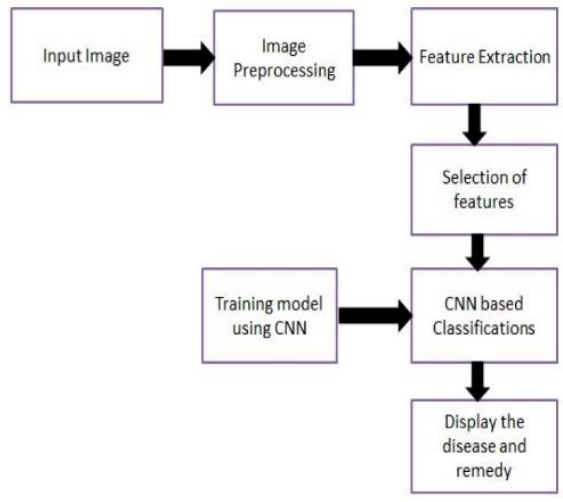

Fig 2: BLOCK DIAGRAM OF CNN [16]

To perform plant disease detection and diagnosis using simple leaves images of healthy and diseased plants Convolutional Neural Network (CNN) models were created, through deep learning methodologies. First user has to capture the plant leaf image from app. The application will send this image to our AI system. The image goes through number of processing steps like preprocessing, feature extraction, selection of feature etc. A novel method of creating a visual database that has been successfully used to train CNN which is a deep residue with $97.8 \%$ accuracy in detecting four species of insects [17].Convolutional neural networks can receive any form of data as input, such as audio, video, images, speech and natural language [18]. CNN constitutes a class of deep, feed forward ANN that has been applied successfully to computer vision applications [19].CNN reached high precision in the large majority of the problems where they have been used, scoring higher precision than other popular image-processing techniques [10].

\section{Conclusion}

Present review study summarize the different applications of artificial intelligence in agriculture sector. The main motive of this study was to brief the applications and available techniques of artificial intelligence to solve the problems of farmers in getting the required yield. The paper also highlights the different literatures, which reflects various methodologies to detect the diseases in crops. From the literature, it is concluded that artificial intelligence is a great tool for a nation's agronomics. Hence, future researchers should organize a proper dataset covering all arena of agriculture and enhance the available technologies to increase the productivity of primary sectors.

\section{Future scope:}

India population is expected to reach more than 1.6 billion by 2030 . With this huge hike in population, one can expect massive demand for agricultural consumption as well. With the advancement in the service sector, there is a big migration of workforce from the primary sector to the tertiary sector. In addition, the ignorance of rising diseases in crops is decreasing the yield of cultivation as well. Food being the primary necessity of human life, future researches need to take direction for reviving the agriculture arena. Artificial Intelligence should be the major tools for the researchers to address the above-mentioned issues. With the great diversity in agronomy species, a detailed database needs to be obtained for various portions of agriculture. By using proper tools of artificial intelligence and with the proper dataset, farming can be made more efficient for farmers. These methods can be considered as the major implementations to solve the future crisis

\section{References}

Tanha Talaviya, Dhara Shah, Nivedita Patel, Manan Shah, "Implementation of artificial intelligence in agriculture for optimisation of irrigation and application of pesticides and herbicides" Volume 4, 2020, Pages 58-73

Anurag Saxena, Truptimayee Suna and Dipankar Saha Regi, “Application of Artificial Intelligence in Indian Agriculture" 2020

Nilay Ganatra and Atul Patel, “A Survey on Diseases Detection and Classification of Agriculture Products using Image Processing and Machine Learning”, International Journal of Computer Applications (0975 - 8887) Volume 180 - No.13, January 2018

Ngozi Clara Eli-Chukwu, "Applications of Artificial Intelligence in Agriculture: A Review”, Engineering, Technology \& Applied Science Research Vol. 9, No. 4, 2019, 4377-4383 
Paras M. Khandelwal and Himanshu Chavhan, "Artificial Intelligence in Agriculture: An Emerging Era of Research”, September 2019

Rajkumar Murugesan , S . K . Sudarsanam , Malathi . G, V. Vijayakumar, Neelanarayanan .V, Venugopal . R , D. Rekha , Sumit Saha, Rahul Bajaj, Atishi Miral , Malolan V, "Artificial Intelligence and Agriculture 5. 0", International Journal of Recent Technology and Engineering (IJRTE) ISSN: 2277-3878, Volume-8 Issue-2, July 2019

Shima Ramesh, Niveditha M, Pooja R, Prasad Bhat N, Shashank N, "Plant Disease Detection Using Machine Learning", April 2018

Mukesh Kumar Tripathi and Dr. Dhananjay D. Maktedar, "Recent Machine Learning Based Approaches for Disease Detection and Classification of Agricultural Products"

Vijay Kumar and Vani K, "Agricultural Robot: Leaf Disease Detection and Monitoring the Field Condition Using Machine Learning and Image Processing”, International Journal of Computational Intelligence Research ISSN 0973-1873 Volume 14, Number 7 (2018), pp. 551-561

A. Kamilaris and F. X. Prenafeta-Boldú, "A review of the use of convolutional neural networks in agriculture" , The Journal of Agricultural Science, June 2018

Chowdhury R. Rahman,* , Preetom S. Arko, Mohammed E. Ali, Mohammad A. Iqbal Khan, Sajid H. Apon, Farzana Nowrin , Abu Wasif, "Identification and recognition of rice diseases and pests using convolutional neural networks", March 2020

Paras M. Khandelwal and Himanshu Chavhan, "Artificial Intelligence in Agriculture: An Emerging Era of Research", September 2019

Yan Guo, Jin Zhang, Chengxin Yin, Xiaonan Hu, Yu Zou, Zhipeng Xue and Wei Wang, "Plant Disease Identification Based on Deep Learning Algorithm in Smart Farming”, Hindawi August 2020

Monishanker Halder, Ananya Sarkar, Habibullah Bahar, "Plant Disease Detection By Image Processing: A Literature Review”, (2019) SDRP Journal of Food Science \& Technology .

Priyanka L. kamble, Anjali C Pise, "Review on Agricultural Plant Disease Detection by using Image Processing" , International Journal of Latest Trends in Engineering and Technology (IJLTET), 2019, ISSN: 2278-621X

Amit Pawar, Mitali Pawaskar, Sonali Ghodke, "Review of Plant Disease Detection and Diagnosis Using Deep Learning Model”, Volume IX, Issue VI, JUNE/2020

H. Liu and J.S. Chahl,, "Proximal detecting invertebrate pestson crops using a deep residual convolutional neural network trained by virtual images",Artificial Intelligence in Agriculture (2021)

Abdel-Hamid O, Mohamed AR, Jiang H, Deng L, Penn G and Yu D, "Convolutional neural networks for speech recognition. IEEE/ACM Transactions on Audio, Speech, and Language Processing” 2014, 22, 1533-1545.

LeCun Y and Bengio Y, "Convolutional networks for images, speech,and time series" In Arbib MA (ed.), The Handbook of Brain Theory and Neural Networks. Cambridge, MA, USA: MIT Press,1995 pp. 255-258.

G. Sambasivam and G. D. Opiyo, A predictive machine learning application in agriculture: Cassava disease detection and classification with imbalanced dataset using convolutional neural networks, Egyptian Informatics Journal, 